\title{
CHATBOTS, CONVERSATIONAL INTERFACES, AND THE STEREOTYPE CONTENT MODEL
}

\author{
Roger Seiler \\ Zurich University of Applied Science \\ roger.seiler@zhaw.ch
}

\author{
Annemarie Schär \\ Zurich University of Applied Science \\ annie.schaer@gmail.com
}

\begin{abstract}
Chatbots are becoming increasingly common in web-based business-to-consumer (B2C) communication. However, this technology can have high failure rates when it comes to correctly processing consumer inquiries, as human interactions and conversational dialogues are complex and highly context dependent. Besides improving the technology behind the chatbots, companies may be able to enhance a client's chatbot experience by using insights from the stereotype content model (SCM). Hence, this paper seeks to find out whether the model can be applied to the domain of chatbots and whether it holds true in this context as well. An online survey was conducted, and the results showed that people perceived the chatbots according to the model. Therefore, companies are advised to use the "loveable star" stereotype in their chatbot communication. Trust did not score significantly higher for this stereotype than for the other SCM stereotypes. This contradicts existing theory and is therefore an invitation for further research.
\end{abstract}

\section{Introduction}

Chatbots can be helpful in booking flights online or in getting assistance when investing money (regarding robo-advisors, see [1] on advising customers when purchasing exchange-traded funds [ETFs] and [2] on how to design interfaces for risk-averse customers). Chatbots are referred to as conversational user interfaces that users can chat with.

Because artificial intelligence (AI) provides humanlike interactions, AI is a key technology for building chatbots that can engage in a dialogue with customers and assist them. It is estimated that chatbots will take over $25 \%$ of customer service communication by 2020 [3]. This estimation is reflected in the current $31.2 \%$ market growth in the United States in 2020 [4]. Therefore, it seems appropriate to take a closer look at how chatbots should be designed as they are the face of a company when a customer communicates with the company or, rather, chats with the company's chatbot.

It is no surprise, then, that in the past years, chatbots have been a top priority for companies, consumers, and researchers alike [5].

This paper will review the literature, derive the research question, and explain the method before describing the hypotheses and analyzing the data. A discussion of the results, limitations, and implications concludes this work.

\section{Literature}

The literature on chatbots has increased (according to Web of Science, $37 \%$ of the publications on chatbots were published in 2019). This increase in attention has led to research on failure rates [6]. System failures can have a large impact on customers' perception. Therefore, the literature on customers' perception of chatbots and how chatbots should be designed is considered here too.

The stereotype content model (SCM) categorizes the judgment of humans in social interactions by rating the interactions regarding two dimensions: warmth (perceived likability) and competence. This model can be applied to social contexts as well [7]-[9]. Failures in chatbot systems are noticed by users and can negatively affect the perceived competence dimension. Competence, in turn, can have positive effects on a chatbot's perceived trust. Portrait pictures of humans can affect the trust perception of chatbots. These two dimensions may be universal dimensions of social perception [8], [10] and are reliable across stimuli, cultures, and time [9]. Therefore, it is no surprise that the SCM also holds true in the context of online insurance sales clerks [11]. Perceived warmth and competence can be embodied in virtual agents as well [12]. A person's perception of the two dimensions can be influenced already by the wording used by a virtual agent [13]. 
Trust can have positive effects on sales [14], [15] and foster customer loyalty [16]. Hence, trust should be included in this study as it positively impacts the dimensions relevant to business success.

Social presence and trust can be modified by the use of avatars [17]. Therefore, avatars play an important role in online communication and can affect customer satisfaction and loyalty [18], two aspects that tend to be critical for business success. Recent studies suggest that anthropomorphic factors play an important role as they can affect social presence [19] and trust [20]. Visual cues [21] and other anthropomorphic design cues show a four times higher effect than functional attributes [22] and can affect credibility [23]. However, high message activity can compensate for the lack of visual cues [24]. Some researchers recommend adding anthropomorphic design cues [25], and we will follow their advice in this work.

\subsection{Trust}

Trust needs to be included and considered as it tends to develop from social interactions (e.g., chatting [26], [27]), visiting websites [14], [15], [28] and online recommendation agents [29], and online purchasing [15], [30], [31]. Trust is further influenced by social presence [20], [32], which in turn is affected by visual representation and identity cues, such as portrait pictures [33]. Especially when the perception of traits, such as warmth and competence, is involved, the topic of trust arises because there is a link between competence and trust [34], [35]. Moreover, one can distinguish between affective and cognitive trust [36]. Hence, trust has emotional as well as rational aspects. Trust not only develops online but also plays a role in virtual environments [12], [34], [37].

Trust itself has a positive impact on consumers' purchase intentions [38], as well as other dimensions (e.g., loyalty [39] and satisfaction [37], [40]) affecting business success, and is therefore important to consider in the design of customer-facing chatbots. Additionally, in conversations with chatbots, trust can play a role as it may be shaped by initial interactions and perceived social presence [41]. Therefore, this construct is included in this study.

\subsection{Stereotype Content Model, Warmth, and Competence}

Reference [10] argues that the warmth dimension relates to good or bad intentions, whereas the competence dimension relates to the ability to fulfill these intentions, with a judgment of the other communication partner being made within seconds. The perception of these two dimensions is an evolutionary-rooted mechanism. The warmth dimension is judged before the competence dimension because it is more important to know whether somebody is friend or foe than whether he or she is capable of executing his or her intentions [10]. The warmth dimension, in turn, affects the perception of trust [42].

\subsection{Research Question}

The use of chatbots is becoming more popular [5], [6], [43] and may be gaining momentum. Because of the COVID-19 pandemic, cutting costs by using chatbots may soon become an attractive option for companies due to rising pressure on cost cutting. The SCM has shown to be valuable in the past in designing website artifacts and customer-facing computer systems that interact with customers. Thus, in this study, we would like to verify whether this model holds true in a chatbot setting, as adding pictures of people to a chatbot may be less costly than improving the technique behind it because merely changing words in chatbot messages can make a difference in person perception [13]. Therefore, this paper seeks to answer the research question of whether the SCM can be applied to the domain of chatbots.

\section{Method}

This study is of a preliminary and exploratory nature. Therefore, complex message interaction and AI implementation of a chatbot are not considered in this work. Adding such complexity with high-level and deep interaction may negatively affect the results. Such an approach would need extensive care regarding both confounding and control variables in order not to jeopardize the research method. Considering these aspects, we decided to use pictures as a starting point because previous research on the use of avatars and pictures as avatars has reported positive effects on social presence [33], trust [44], [45], and competence [45]. Furthermore, interfaces with avatars are perceived as more likable [46]. The stimulus material (pictures for each SCM stereotype) has been generated and verified in a previous study [11]. Therefore, this material was used in this study to verify whether the SCM and the stimulus material of the two stereotypes-Chatbot 1 ("lovable star"; see Figure 1), rating high on the competence and warmth dimensions, and Chatbot 2 ("incompetent jerk"; see Figure 2), scoring low on the warmth and competence dimensionswork in the context of chatbots too. Recent studies have pointed out that in a highly interactive and conversational setting, high message activity can compensate for the lack of visual cues [24]. Visual design also plays an important role, as it can affect social presence as well as trust [20]. Visual cues [21] and other anthropomorphic 
design cues show a four times higher effect than functional attributes [22] and can affect credibility [23].

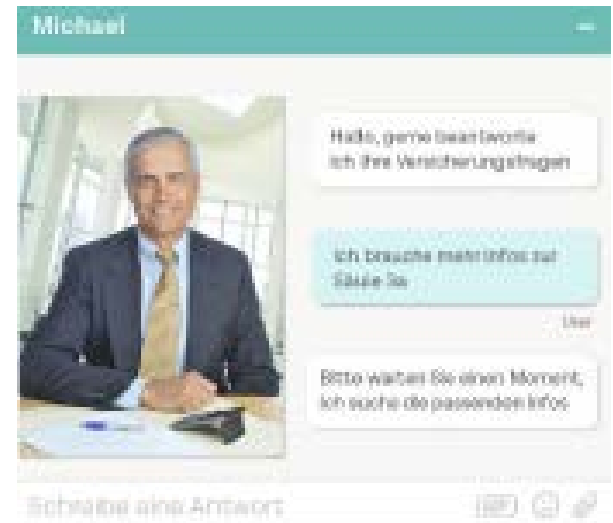

Figure 1. Chatbot 1: "Lovable Star"

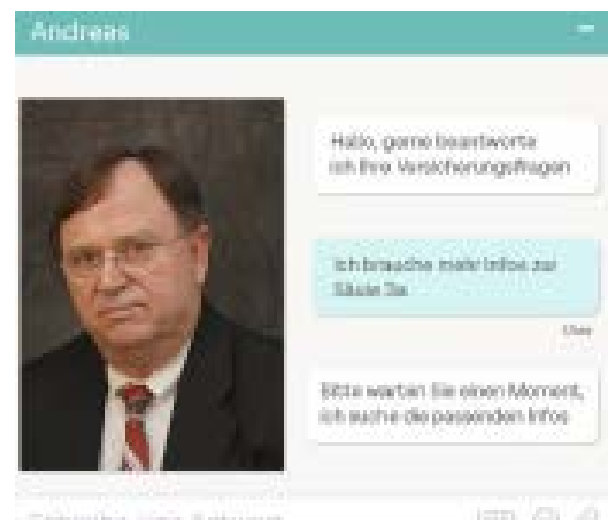

Figure 2. Chatbot 2: "Incompetent Jerk"

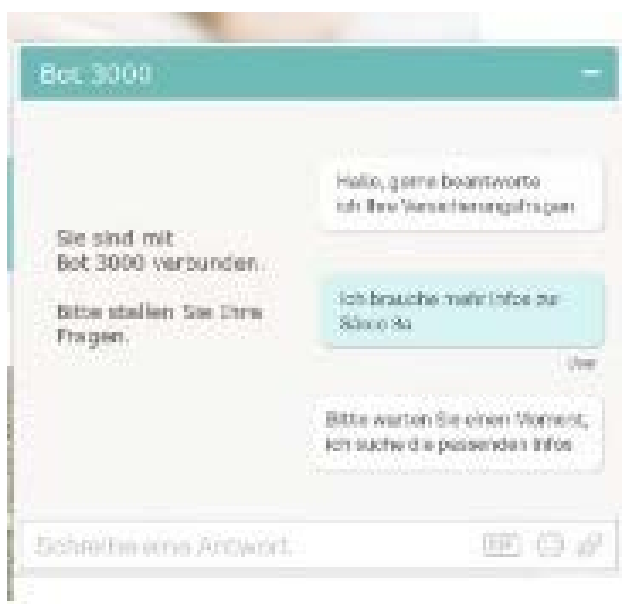

Figure 3. Chatbot 3: Control Group
Therefore, adding images to chatbots seems a valid approach to testing the SCM in the chatbot context. Screenshots were integrated into an online survey to ensure that every participant is shown the same interaction (text dialogue) and to verify the SCM. In this context, the mere perception of chatbots (the dimensions are judged instantly; see Section 2.2) should work and therefore show effects. Hence, the verification of the model should be possible with this research design. Not only does this approach not overcomplicate the technical setup or potentially add confounding variables, but it is also adequate for conducting initial research on the topic in this domain and context. Moreover, to prevent overloading the online survey, this initial study uses the two stereotypes that rank highest and lowest on both the warmth and the competence dimension instead of adding all four stereotypes. A third group (Chatbot 3), the control group, was implemented without a picture (see Figure 3).

A control question was asked, and a manipulation check was conducted, to ensure that the participants took notice of the chatbot, and they were randomly assigned to one of the three groups in the online questionnaire.

Ice breaker questions regarding participants' sociodemographic backgrounds were asked in the first stage of the online survey. Subsequently, the warmth/likability and competence scales and the trust scale from [47] were used, making this study comparable to prior research in the field.

Semantic differentials regarding the dimensions, as well as Likert scales for metric measurements, were used, both seven-point and multiple-item, as this approach tends to have an advantage over single-item operationalization [48].

\subsection{Hypotheses}

In line with existing theory regarding the SCM [8], [10], [42], the lovable star chatbot should be perceived as more competent and warm than Chatbot 2 (incompetent jerk) and Chatbot 3 (control), hence hypotheses (Hs) 1, 2, 3, and 4 (see Table 1). Competence relates to trust as its perception [36], [49], [50] positively influences trust, so the lovable star chatbot should be perceived as more trustworthy than Chatbot 2 (H5) and Chatbot 3 (H6). An overview of hypotheses one to six is given in Table 1 as well as the decision of acceptance or rejection. 
Table 1: Overview of the Hypotheses

\begin{tabular}{|c|l|r|}
\hline$\#$ & \multicolumn{1}{|c|}{ Text } & Result \\
\hline 1 & $\begin{array}{l}\text { Chatbot 1 (lovable star design) is } \\
\text { perceived as warmer than Chat- } \\
\text { bot 2 (incompetent jerk design) }\end{array}$ & Accept \\
\hline 2 & $\begin{array}{l}\text { Chatbot 1 (lovable star design) is } \\
\text { perceived as more competent } \\
\text { than Chatbot 2 (incompetent jerk } \\
\text { design) }\end{array}$ & Accept \\
\hline 3 & $\begin{array}{l}\text { Chatbot 1 (lovable star design) is } \\
\text { perceived as warmer than Chat- } \\
\text { bot 3 (simple text chatbot) }\end{array}$ & Accept \\
\hline 4 & $\begin{array}{l}\text { Chatbot 1 (lovable star design) is } \\
\text { perceived as more competent } \\
\text { than Chatbot 3 (simple text chat- } \\
\text { bot) }\end{array}$ & Accept \\
\hline 5 & $\begin{array}{l}\text { Chatbot 1 (lovable star design) is } \\
\text { perceived as more trustworthy } \\
\text { than Chatbot 2 (incompetent jerk } \\
\text { design). }\end{array}$ & Accept \\
\hline 6 & $\begin{array}{l}\text { Chatbot 1 (lovable star design) is } \\
\text { perceived as more trustworthy } \\
\text { than Chatbot 3 (simple text bot). }\end{array}$ & Reject \\
\hline
\end{tabular}

\section{Results, Discussion, Implications, and Limitations}

Data collection was conducted between April 24, 2018, and May 3, 2018, by email. A total of 140 participants fully completed the online questionnaire (Group 1: $n=46$; Group 2: $n=44$; and Group 3: $n=50$ ).

\subsection{Sample Description}

Regarding gender, the sample is composed of $46.5 \%$ women, $51.2 \%$ men, and $2.3 \%$ not wishing to state their gender identity. The age ranges from 19 to 54 years, with a mean of 27.41 . Of the participants, $87.6 \%$ are single, $8.5 \%$ married, $1.6 \%$ divorced, and $2.3 \%$ did not prefer to say.

\subsection{Data Analysis}

IBM SPSS Statistics 24 was used. As the number of participants in each group exceeds thirty, a normal distribution may be assumed, so parametric hypothesis testing was conducted.

First, Cronbach's alpha for each construct was calculated to test the reliability of the scales. All values in Table 2 meet the minimal value of .7 and are above Nunally's threshold [51]. The sample size per group is, according to the central limit theorem, large enough.
This allows metric testing to be used, and $t$ tests were conducted to test the hypotheses.

The warmth scores are significantly higher for Chatbot 1 than for Chatbot 2 (see Table 3), so H1 is accepted. Warmth is higher for Chatbot 1 than for Chatbot 3 , and therefore H3 is also accepted.

Regarding competence, Chatbot 1 shows higher values than Chatbot 2, leading to the acceptance of $\mathrm{H} 2$. Competence is also perceived as higher for Chatbot 1 than for Chatbot 3, thus supporting H4.

Trust scores were compared between Chatbots 1 and 2 , showing significant effects in favor of Chatbot 1 , which is perceived as more trustworthy than Chatbot 2. Therefore, $\mathrm{H} 5$ is accepted.

However, Chatbot 1 is not perceived as more trustworthy than Chatbot 3, and therefore H6 is rejected.

Table 2: Reliability Measures

\begin{tabular}{|l|c|}
\hline \multicolumn{1}{|c|}{ Construct } & $\begin{array}{c}\text { Cronbach's } \\
\text { alpha }\end{array}$ \\
\hline Warmth (Chatbot 1) & .823 \\
\hline Warmth (Chatbot 2) & .824 \\
\hline Warmth (Chatbot 3) & .777 \\
\hline Competence (Chatbot 1) & .942 \\
\hline Competence (Chatbot 2) & .916 \\
\hline Competence (Chatbot 3) & .925 \\
\hline Trust (Chatbot 1) & .917 \\
\hline Trust (Chatbot 2) & .940 \\
\hline Trust (Chatbot 3) & .874 \\
\hline
\end{tabular}

Table 3: Group Comparison

\begin{tabular}{|l|c|c|c|c|}
\hline Construct & $\boldsymbol{M}$ & SD & $\boldsymbol{t}$ & $\boldsymbol{p}$ \\
\hline Warmth (1) & 4.59 & 1.57 & - & - \\
\hline Warmth (2) & 3.13 & 1.39 & 8.72 & $* * *$ \\
\hline Warmth (3) & 3.82 & 1.60 & 4.51 & $* * *$ \\
\hline Competence (1) & 5.01 & 1.45 & - & - \\
\hline Competence (2) & 4.29 & 1.56 & 4.72 & $* * *$ \\
\hline Competence (3) & 3.97 & 1.43 & 7.52 & $* * *$ \\
\hline Trust (1) & 4.51 & 1.58 & & \\
\hline Trust (2) & 4.15 & 1.59 & 2.25 & $*$ \\
\hline Trust (3) & 4.43 & 1.30 & .599 & n.s. \\
\hline \multicolumn{2}{|l|}{$* * *<.001, * *<.01, *<.05$, and n.s. $>.05$} \\
\hline
\end{tabular}

\subsection{Discussion}

On the basis of the data analysis and $t$ tests, the warmth and competence dimensions are significantly higher for the lovable star stereotype (highest mean value of all three chatbots) than for the incompetent jerk stereotype. Therefore, we can conclude that both the stimulus material and the SCM hold true in the context of chatbots. In other words, the participants judged the chatbots in accordance with the SCM. 
The participants showed higher trust levels for the lovable star stereotype than for the incompetent jerk stereotype. Nevertheless, it must be pointed out that this does not hold true when comparing the lovable star stereotype with Chatbot 3 without a picture. This is interesting as prior research and theory suggest that a chatbot with a picture as social presence should show higher trust values than a chatbot without a picture [52]. Presumably, the judgment of cues regarding the dimensions warmth and competence is more important because they are more prominently displayed.

One possible explanation could be rooted in the processing of information. If a higher cognitive load is needed - as may be the case in the context of Chatbot 3 because the picture and thus visual cues are missing-a higher level of trust may be generated. A higher amount of internal cognitive information processing may be seen as more credible than relying on external visual cues.

According to some models of information processing, such as the elaboration likelihood model (ELM) [53], [54], with a central component (critical thinking and intense information processing) and a peripheral component (relying on simple cues, e.g., pictures), the missing picture may lead to higher cognitive engagement. Thus, this may garner more trust as internal information and relying upon it may lead to higher trust levels.

However, these aspects are just lines of thought, and further research is needed to elaborate on them and gain additional insights and evidence.

\subsection{Implications}

As discussed above, one can build upon our results and advise companies that consider using a customerfacing chatbot for their interactions with customers. Companies may choose avatars or pictures for their interfaces in accordance with the SCM model. This can help build trust, which, in turn, has positive effects on purchase intentions [28], [37], [38], [41], repurchase and recommendation intentions, and satisfaction [37], [40]. All these aspects are business relevant and can positively impact business success.

Moreover, our research suggests that regarding warmth and competence, chatbots with pictures of people are perceived as warmer and more competent. Therefore, companies intending to signal competence or warmth to their online customers, as is typically the case for insurance companies, are advised to use chatbots with pictures.

\subsection{Limitations}

The pictures of the chatbots used in the online survey were of course limited in their functionality (no chatbot interaction), and this must be taken into account when interpreting the data and results.

Additionally, the stimulus material is gender specific, and the results may be different when other stimuli showing female sales representatives are used.

This study was conducted with a sample composed predominantly of student participants, and the results may not be the same for a representative sample of the population and may also vary between countries and cultures.

\section{Further Research}

As this study used only two out of four stereotypes, future work may use all four stereotypes to verify whether consumers differentiate further and whether the implications of the SCM hold true for all four stereotypes. Therefore, adding the two other stereotypes, incompetent jerk (low on competence and warmth) and competent jerk (high on competence and low on warmth), would complete the stereotypes and expand the results of our work.

In the past, profession groups have been investigated [55], and this could also be done in the context of chatbots. Because pictures of a dialogue with the chatbot were used, follow-up research may implement an interactive version of this online survey. This would enable testing how much impact a picture has in comparison with other elements, such as messaging or full chatbot interaction.

Gender may be an additional line of research as our stimulus material consisted only of male sales representatives. Verifying our results regarding gender is important because past work on gender [56] and robots [57] suggests that gender may play a role in person perception. Furthermore, shedding light on ethnicity may lead to a holistic view, as it may impact the presented results [55] and support gender and ethnicity equality.

Culture, too, may be worthwhile investigating further as it is not clear whether these results regarding the SCM are valid across different regions and cultures.

Future research may also want to explore whether the SCM applies to a chatbot with a 3D representation or even with an interactive virtual assistant so that the role of immersion and virtual reality could be investigated.

Finally, future research may be following up on the use of the SCM and the implications this may have for the perception of trust. Therefore, risk analysis may be worthwhile investigating further. 


\section{Concluding Remarks}

The results of this study suggest that the SCM is also valid in the context of chatbots. Accordingly, companies interested in having virtual agents, such as chatbots, that need to be perceived as warm and competent are advised to use pictures that are in line with the SCM.

The use of pictures is advised in situations in which these two dimensions are relevant (e.g., insurance clerks), as a chatbot with images showed higher values of warmth and competence than a chatbot without a visual key. The use of pictures is also advised from the viewpoint of human perception, as initial judgment takes place instantly and pictures can help customers judge the warmth and competence of a chatbot immediately.

The results do not suggest that the use of pictures leads to higher levels of trust compared with the control (text chatbot). Further research is needed as to why this contradicts the SCM but only in comparison with a text chatbot that has lower levels of warmth and competence.

\section{References}

[1] D. Jung, V. Dorner, F. Glaser, und S. Morana, «RoboAdvisory: Digitalization and Automation of Financial Advisory», Business \& Information Systems Engineering, Bd. 60, Nr. 1, S. 81-86, Feb. 2018, doi: 10.1007/s12599018-0521-9.

[2] D. Jung, V. Dorner, C. Weinhardt, und H. Pusmaz, «Designing a robo-advisor for risk-averse, low-budget consumers», Electronic Markets, Bd. 28, Nr. 3, S. 367-380, Aug. 2018, doi: 10.1007/s12525-017-0279-9.

[3] Gartner, «Gartner Says 25 Percent of Customer Service Operations Will Use Virtual Customer Assistants by 2020», Gartner, Feb. 19, 2018. https://www.gartner.com/en/newsroom/press-releases/2018-02-19-gartner-says-25-percent-of-customer-service-operations-willuse-virtual-customer-assistants-by-2020.

[4] M.-H. Nguyen, «Chatbot Market 2020: Stats, Trends, Size and Ecosystem Research - Business Insider», Business Insider, Jan. 23, 2020. https://www.businessinsider.com/chatbot-market-statstrends? $\mathrm{r}=\mathrm{US} \& \mathrm{IR}=\mathrm{T}$ (zugegriffen Juli 03, 2020).

[5] R. Dale, «The return of the chatbots», Natural Language Engineering, Bd. 22, Nr. 05, S. 811-817, Sep. 2016, doi: 10.1017/S1351324916000243.

[6] W. Knight, «How to Prevent a Plague of Dumb Chatbots | MIT Technology Review», MIT Technology Review, Apr. 18, 2016. https://www.technologyreview.com/2016/04/18/160940/how-to-prevent-aplague-of-dumb-chatbots/ (zugegriffen Juli 03, 2020).

[7] T. Casciaro und M. S. Lobo, «Competent jerks, lovable fools, and the formation of social networks.», Harvard business review, Bd. 83, Nr. 6, S. 92-9, 149, Juni 2005, doi: 10.1037.

[8] A. J. C. Cuddy, S. T. Fiske, und P. Glick, Warmth and Competence as Universal Dimensions of Social
Perception: The Stereotype Content Model and the BIAS Map, Bd. 40. 2008.

[9] S. T. Fiske, A. J. C. Cuddy, P. Glick, und J. Xu, «A model of (often mixed) stereotype content: Competence and warmth respectively follow from perceived status and competition.», Journal of Personality and Social Psychology, Bd. 82, Nr. 6, S. 878-902, 2002, doi: 10.1037/00223514.82.6.878.

[10] S. T. Fiske, A. J. C. Cuddy, und P. Glick, «Universal dimensions of social cognition: warmth and competence», Trends in Cognitive Sciences, Bd. 11, Nr. 2, S. 77-83, Feb. 2007, doi: 10.1016/j.tics.2006.11.005.

[11] R. Seiler, J. J. Hari, und S. Kavci, «Changing the warmth and competence dimensions: experimental validation in the context of insurance consultants», in Academy of Marketing Conference 2015, Limerick, 7-9 July 2015, 2015, S. 1-9.

[12] V. Demeure, R. Niewiadomski, und C. Pelachaud, «How is believability of a virtual agent related to warmth, competence, personification, and embodiment?», Presence: teleoperators and virtual environments, Bd. 20, Nr. 5, S. 431-448, 2011.

[13] M. V. Garrido, S. Godinho, und G. R. Semin, «The "ins" and "outs" of person perception: The influence of consonant wanderings in judgments of warmth and competence», Journal of Experimental Social Psychology, Bd. 82, S. 1-5, Mai 2019, doi: 10.1016/j.jesp.2018.11.004.

[14] D. J. Kim, D. L. Ferrin, und H. R. Rao, «A trust-based consumer decision-making model in electronic commerce: The role of trust, perceived risk, and their antecedents», Decision Support Systems, Bd. 44, Nr. 2, S. 544 564, Jan. 2008, doi: 10.1016/j.dss.2007.07.001.

[15] Y. Kim und R. A. Peterson, «A Meta-analysis of Online Trust Relationships in E-commerce», Journal of Interactive Marketing, Bd. 38, S. 44-54, Mai 2017, doi: 10.1016/j.intmar.2017.01.001.

[16] D. Cyr, K. Hassanein, M. Head, und A. Ivanov, «The role of social presence in establishing loyalty in e-Service environments», Interacting with Computers, Bd. 19, Nr. 1, S. 43-56, Jan. 2007, doi: 10.1016/j.intcom.2006.07.010.

[17] R. Etemad-Sajadi, «The impact of online real-time interactivity on patronage intention: The use of avatars», Computers in Human Behavior, Bd. 61, S. 227-232, Aug. 2016, doi: 10.1016/j.chb.2016.03.045.

[18] M. Holzwarth, C. Janiszewski, und M. M. Neumann, «The Influence of Avatars on Online Consumer Shopping Behavior», Journal of Marketing, Bd. 70, Nr. 4, S. 19-36, Okt. 2006, doi: 10.1509/jmkg.70.4.019.

[19] T. Araujo, «Living up to the chatbot hype: The influence of anthropomorphic design cues and communicative agency framing on conversational agent and company perceptions», Computers in Human Behavior, Bd. 85, S. 183-189, Aug. 2018, doi: 10.1016/j.chb.2018.03.051.

[20] D.-C. Toader $u$. a., «The Effect of Social Presence and Chatbot Errors on Trust», Sustainability, Bd. 12, Nr. 1, Art. Nr. 1, Jan. 2020, doi: 10.3390/su12010256.

[21] N. Wuenderlich und S. Paluch, «A Nice and Friendly Chat with a Bot: User Perceptions of AI-Based Service Agents», ICIS 2017 Proceedings, Dez. 2017, [Online]. 
Verfügbar unter: https://aisel.aisnet.org/icis2017/ServiceScience/Presentations/11.

[22] T. Rietz, I. Benke, und A. Maedche, «The Impact of Anthropomorphic and Functional Chatbot Design Features in Enterprise Collaboration Systems on User Acceptance», Wirtschaftsinformatik 2019 Proceedings, Feb. 2019, [Online]. Verfügbar unter: https://aisel.aisnet.org/wi2019/track13/papers/7.

[23] K. L. Nowak und C. Rauh, «The Influence of the Avatar on Online Perceptions of Anthropomorphism, Androgyny, Credibility, Homophily, and Attraction», J Comput Mediat Commun, Bd. 11, Nr. 1, S. 153-178, Nov. 2005, doi: 10.1111/j.1083-6101.2006.tb00308.x.

[24] E. Go und S. S. Sundar, «Humanizing chatbots: The effects of visual, identity and conversational cues on humanness perceptions», Computers in Human Behavior, Bd. 97, S. 304-316, Aug. 2019, doi: 10.1016/j.chb.2019.01.020.

[25] M. Adam, M. Wessel, und A. Benlian, «AI-based chatbots in customer service and their effects on user compliance», Electron Markets, März 2020, doi: 10.1007/s12525-020-00414-7.

[26] J. Zheng, N. Bos, J. S. Olson, und G. M. Olson, «Trust without touch: Jump-start trust with social chat», in CHI'01 extended abstracts on Human factors in computing systems, 2001, S. 293-294, doi: 10.1145/634067.634241.

[27] J. Zheng, E. Veinott, N. Bos, J. S. Olson, und G. M. Olson, «Trust without Touch: Jumpstarting long-distance trust with initial social activties», in CHI 2002 Proceedings, April 20-25, Minneapolis, Minnesota, USA, 2002, Bd. 4, S. 141-146.

[28] P. Beatty, I. Reay, S. Dick, und J. Miller, «Consumer Trust in e-Commerce Web Sites: A Meta-study», ACM Comput. Surv., Bd. 43, Nr. 3, S. 14:1-14:46, Apr. 2011, doi: $10.1145 / 1922649.1922651$.

[29] P. A. Dabholkar und X. Sheng, «Consumer participation in using online recommendation agents: effects on satisfaction, trust, and purchase intentions», Service Industries Journal, Bd. 32, Nr. 9, S. 1433-1449, 2012, doi: 10.1080/02642069.2011.624596.

[30] H. H. Chang und S. W. Chen, «The impact of online store environment cues on purchase intention: Trust and perceived risk as a mediator», Online Information Review, Bd. 32, Nr. 6, S. 818-841, Nov. 2008, doi: 10.1108/14684520810923953.

[31] J. Tang, H. Gao, H. Liu, und A. Das Sarma, «eTrust: Understanding Trust Evolution in an Online World», in Proceedings of the 18th ACM SIGKDD International Conference on Knowledge Discovery and Data Mining, New York, NY, USA, 2012, Bd. Beijing, C, S. 253-261, [Online]. Verfügbar unter: http://doi.acm.org/10.1145/2339530.2339574.

[32] C. R. De, S. S. C. e, und F. R. Alparone, «Millennials’ attitude toward chatbots: an experimental study in a social relationship perspective», International Journal of Retail \& Distribution Management, Bd. ahead-of-print, Nr. ahead-of-print, Jan. 2020, doi: 10.1108/IJRDM-122019-0406.

[33] C. S. Oh, J. N. Bailenson, und G. F. Welch, «A Systematic Review of Social Presence: Definition, Antecedents, and Implications», Frontiers in Robotics and AI, Bd. 5, Okt. 2018, doi: 10.3389/frobt.2018.00114.

[34] T. Kugler, B. Ye, D. Motro, und C. N. Noussair, «On Trust and Disgust: Evidence From Face Reading and Virtual Reality», Social Psychological and Personality Science, S. 194855061985630, Juli 2019, doi: $10.1177 / 1948550619856302$.

[35] D. Yang und M. J. Wu, «Does Customer Trust Play a Mediating Role Between Salesperson Competence and Performance ?», International Journal of Management, Economics and Social Sciences, Bd. 3, Nr. 2, S. 100-121, 2014.

[36] D. J. McAllister, «Affect- and Cognition-Based Trust as Foundations for Interpersonal Cooperation in Organizations», Academy of Management Journal, Bd. 38, Nr. 1, S. 24-59, Feb. 1995, doi: 10.5465/256727.

[37] P. Papadopoulou, «Applying virtual reality for trustbuilding e-commerce environments», Virtual Reality, Bd. 11, Nr. 2-3, S. 107-127, Juni 2007, doi: 10.1007/s10055006-0059-x.

[38] B. Lu, W. Fan, und M. Zhou, «Social presence, trust, and social commerce purchase intention: An empirical research», Computers in Human Behavior, Bd. 56, S. 225237, 2016, doi: 10.1016/j.chb.2015.11.057.

[39] A. Bilgihan, «Gen Y customer loyalty in online shopping: An integrated model of trust, user experience and branding», Computers in Human Behavior, Bd. 61, S. 103-113, Aug. 2016, doi: 10.1016/j.chb.2016.03.014.

[40] G. Das, «Antecedents and consequences of trust: an e-tail branding perspective», Intl J of Retail \& Distrib Mgt, Bd. 44, Nr. 7, S. 713-730, Juli 2016, doi: 10.1108/IJRDM-062015-0089.

[41] A. Ogonowski, A. Montandon, E. Botha, und M. Reyneke, «Should new online stores invest in social presence elements? The effect of social presence on initial trust formation», Journal of Retailing and Consumer Services, Bd. 21, Nr. 4, S. 482-491, 2014, doi: 10.1016/j.jretconser.2014.03.004.

[42] A. J. C. Cuddy, P. Glick, und A. Beninger, «The dynamics of warmth and competence judgments, and their outcomes in organizations», Research in Organizational Behavior, Bd. 31, Nr. 0, S. 73-98, 2011, doi: http://dx.doi.org/10.1016/j.riob.2011.10.004.

[43] M. F. McTear, «The Rise of the Conversational Interface: A New Kid on the Block?», in Future and Emerging Trends in Language Technology. Machine Learning and Big Data, Bd. 10341, J. F. Quesada, F.-J. Martín Mateos, und T. López Soto, Hrsg. Cham: Springer International Publishing, 2017, S. 38-49.

[44] U. Steinbrück, H. Schaumburg, S. Duda, und T. Krüger, "A Picture Says More Than A Thousand Words - Photographs As Trust Builders in E-Commerce Websites», S. 2.

[45] W. Wang, L. Qiu, D. Kim, und I. Benbasat, «Effects of rational and social appeals of online recommendation agents on cognition- and affect-based trust», Decision Support Systems, Bd. 86, S. 48-60, Juni 2016, doi: 10.1016/j.dss.2016.03.007.

[46] J. Lester, S. Towns, C. Calloway, und P. FitzGerald, «Deictic and emotive communication in animated pedagogical agents», in Embodied Conversational Agents, Boston, Massachusetts: MIT Press, 2000, S. 123-154. 
[47] M.-A. Reinhard, M. Messner, und S. L. Sporer, «Explicit Persuasive Intent and Its Impact on Success at Persuasion-The Determining Roles of Attractiveness and Likeableness», Journal of Consumer Psychology, Bd. 16, Nr. 3, S. 249-259, Jan. 2008, doi: 10.1207/s15327663jcp1603_7.

[48] A. Diamantopoulos, M. Sarstedt, C. Fuchs, P. Wilczynski, und S. Kaiser, «Guidelines for choosing between multi-item and single-item scales for construct measurement: a predictive validity perspective», Journal of the Academy of Marketing Science, Bd. 40, Nr. 3, S. 434449, Mai 2012, doi: 10.1007/s11747-011-0300-3.

[49] E. P. Becerra und V. Badrinarayanan, «The influence of brand trust and brand identification on brand evangelism», Jnl of Product \& Brand Mgt, Bd. 22, Nr. 5/6, S. 371-383, Aug. 2013, doi: 10.1108/JPBM-09-2013-0394.

[50] H. D. McKnight, V. Choudhury, und C. Kacmar, «The impact of initial consumer trust on intentions to transact with a web site: a trust building model», The Journal of Strategic Information Systems, Bd. 11, Nr. 3, S. 297-323, Dez. 2002, doi: 10.1016/S0963-8687(02)00020-3.

[51] J. Nunally, Psychometric theory. New York: McGrawHill, 1978.

[52] L. Qiu und I. Benbasat, «Online consumer trust and live help interfaces: The effects of text-to-speech voice and three-dimensional avatars», International Journal of Human-Computer Interaction, Bd. 19, Nr. 1, S. 75-94, 2005, doi: 10.1207/s15327590ijhc1901_6.

[53] J. T. Cacioppo und R. E. Petty, «The elaboration likelihood model of persuasion", ACR North American Advances, 1984.

[54] R. E. Petty und J. T. Cacioppo, «Source factors and the elaboration likelihood model of persuasion», ACR North American Advances, 1984.

[55] R. Imhoff, J. Woelki, S. Hanke, und R. Dotsch, «Warmth and competence in your face! Visual encoding of stereotype content.», Frontiers in psychology, Bd. 4, Nr. June, S. 386, Jan. 2013, doi: 10.3389/fpsyg.2013.00386.

[56] D. Cyr und C. Bonanni, «Gender and website design in e-business», International Journal of Electronic Business, Bd. 3, Nr. 6, S. 565-582, 2005.

[57] B. Tay, Y. Jung, und T. Park, «When stereotypes meet robots: The double-edge sword of robot gender and personality in human-robot interaction", Computers in $\mathrm{Hu}$ man Behavior, Bd. 38, S. 75-84, Sep. 2014, doi: 10.1016/j.chb.2014.05.014. 